\title{
Optimal preparation of water samples for the examination of colloidal material by transmission electron microscopy
}

\author{
Charles-Philippe Lienemann', Andreas Heissenberger ${ }^{2}$, Gary Grant Leppard ${ }^{3}$, \\ Didier Perret ${ }^{1, *}$
}

\author{
'Institute of Inorganic and Analytical Chemistry, University of Lausanne, CH-1015 Lausanne, Switzerland \\ ${ }^{2}$ Institute of Zoology, Department of Marine Biology, University of Vienna, Althanstrasse 14, A-1090 Vienna, Austria \\ ${ }^{3}$ National Water Research Institute, Canada Centre for Inland Waters, Burlington, Ontario, Canada L7R 4A6
}

\begin{abstract}
We describe an effective combination of techniques for the analysis of aquatic colloidal material by transmission electron microscopy (TEM). Specimens are prepared either by direct and quantitative ultracentrifugation onto supporting grids (whole mounts approach) or by resin embedding and ultramicrotomy (thin sections approach). Both preparation schemes require fastidious attention to protocol to obtain minimally perturbed images of native colloidal material present in natural samples. In the case of the whole mounts approach, 'optical properties' (turbidity, light scattering, fluorimetry) of the suspension are measured in order to evaluate the optimal specimen preparation according to a dataset of optimal centrifugation conditions. This fast and non-artifacted approach is complementary to the time-consuming thin sections approach, their combination allowing a clear representation of the ultrastructure of colloids and their 3-dimensional relationships. The whole mounts permit quantification of relative amounts of different colloid 'species' while the sections permit colloid descriptions according to internal differentiation. The use of this correlative approach is illustrated with lacustrine and marine samples, with a special emphasis on the interactions between bacteria, their fibrillar extracellular polymeric substances (fibrils, exopolymers) and other colloids.
\end{abstract}

KEY WORDS: Transmission electron microscopy (TEM) - Ultracentrifugation - Resin embedding · Thin sectioning $\cdot$ Exopolymers $\cdot$ Bacteria $\cdot$ Mineral colloids

\section{INTRODUCTION}

A high number and ubiquitous occurrence of aquatic colloids have recently been demonstrated for both marine (Koike et al. 1990) and freshwater ecosystems (Leppard 1992a, Perret et al. 1994). The classification of colloids and assignation of their activities are in progress (Leppard et al. 1997); of special interest are attempts to characterize native colloid associations (Filella et al. 1993, Buffle \& Leppard 1995, Pizarro et al. 1995, Leppard et al. 1996). There is a focus on those colloids that influence biological impact on aquatic ecosystems by contributing to heterogeneous aggre-

\footnotetext{
- Addressee for correspondence

E-mail: didier.perret@icma.unil.ch
}

gate formation (Heissenberger et al. 1996a, b, Liss et al. 1996). To further these goals, this paper presents an optimal correlative preparatory methodology for the examination of aquatic colloidal material by transmission electron microscopy (TEM). For case studies, it considers water samples relevant to specific biological phenomena from both a marine and a freshwater ecosystem.

TEM has been shown to be an effective tool for the visualization and characterization of colloidal organic matter, and the only tool permitting analyses on a 'per particle' basis at near nanometer resolution. However, conventional preparation schemes for TEM specimens usually require chemical stabilization, followed by sample dehydration and resin embedding, prior to ultramicrotomy. The multiple steps can lead to mor- 
phological artifacts, especially if extraction and dehydration are not controlled (Leppard et al, 1996, 1997). As an alternative and complementary specimen preparation technique, ultracentrifugation has been used to study aquatic colloids from many ecosystems, including the submicrometer particles of soils (Nomizu \& Mizuike 1986, Perret et al. 1991, 1994, Wells \& Goldberg 1992, Mavrocordatos \& Perret 1995). The minimally perturbing protocols among them have led to realistic whole mount specimens having the advantages of rapidity, simplicity of operation, reproducibility and the possibility of particle quantification by counting or image analysis.

When using an ultracentrifuged water sample to study associations and interactions between aquatic colloids with TEM, it is essential that the specimen plane be neither overcovered with colloids (leading to biased conclusions) nor undercovered (increasing the time and cost factors). To avoid inappropriate coverage, one must have a capacity to estimate in advance the number concentration of colloids in the sample and to prepare specimens for quantification accordingly. By gaining advance knowledge on the optimal loading of colloids onto TEM grids, one can avoid costly backand-forth work (field to laboratory to TEM and back again).

In this study we demonstrate that the 'optical properties' of a sample (determined with a photometer, turbidimeter or fluorimeter) can be adequately explored to prepare whole mounts for quantitative analysis by TEM. Used correlatively with the more classical embedding and ultramicrotomy approach, whole mount specimens provide a degree of quantification which is usually cost-prohibitive for thin section analyses, while allowing one to relate the internal details of colloids derived from sections to the corresponding colloids quantified by whole mounts. Also in this study, we show how the improved technology is applied to water samples rich in fibrils having biological impact on natural processes. When a certain level of detail is achieved by a correlative approach, one can then switch to the ultracentrifugation method alone for systematic quantitative research.

\section{MATERIAL AND METHODS}

Fig, 1a schematizes the complete methodology (whole mounts approach and thin sections approach; correlative electron microscopy) that can lead to a realistic representation of the morphology and associations between colloids in natural waters. Fig. $1 \mathrm{~b}$ shows the specimen preparation scheme by ultracentrifugation developed for the TEM examination of suspended material, including living and non-living particles. The method was developed and validated with synthetic hematite spheres, and then checked with freshwater samples prior to being applied to marine systems. Each step of both approaches is described in detail below.

Standardization of the whole mounts approach. Prior to use of the proposed scheme, the complete ultracentrifugation procedure was standardized with known suspensions of homodispersed hematite microparticles $\left(\alpha-\mathrm{Fe}_{2} \mathrm{O}_{3}\right.$, diameter $=60 \mathrm{~nm}$, density $=5.24 \mathrm{~g} \mathrm{~cm}^{-3}$, Matijevic \& Scheiner 1978). The applicability of the procedure was checked with river water samples and the procedure was then applied to lacustrine and marine samples to quantitatively collect optimal and representative amounts of particulate exopolymeric material onto TEM grids.

Standard suspensions containing known mass concentrations of hematite $\left(10^{1}\right.$ to $\left.10^{3} \mu \mathrm{g} \mathrm{l}^{-1}\right)$ were prepared in slightly acidic $\left([\mathrm{HCl}]=10^{-4} \mathrm{M}\right)$ ultrapure water (Elgastat UHQ-II) to avoid coalescence of microspheres $\left(\mathrm{pH}_{z p c}=8.5\right.$; Liang 1988). The 'optical properties' of these suspensions, determined in our case as intensity of scattered light (measured by a fluorimeter with excitation and emission monochromators set to zero order; Perkin-Elmer LS50 fluorimeter) or as turbidity (Hach Turbidimeter $2100 \mathrm{~N}$ ) were measured in order to build the first calibration curve (see Fig. 2).

Different volumes of the previous suspensions, ranging from 2 to $20 \mathrm{ml}$, were ultracentrifuged (28 $000 \mathrm{rpm}$, $\mathrm{RCF}=135000 \times g, 1 \mathrm{~h}, 10^{\circ} \mathrm{C}$; Kontron Centrikon T2070 equipped with a TST-28.38 swing-out rotor) above grids (see below) which were further examined and micrographed by TEM. In order to build the second calibration curve, the characteristics of the particles, determined in our case as the total cross-section area of hematite particles per unit analyzed surface area, were measured on micrographs by image analysis (Samba, Alcatel TITN) as a function of the total mass of hematite present in the volume of centrifuged suspension (see Fig. 3)

Sampling of natural waters for the whole mounts and thin sections approaches. For the control of the proposed ultracentrifugation method, river water (Arve River, Geneva, Switzerland) was sampled during flood and low water events. During flood conditions, water was sedimented $\left(2 \mathrm{~h}, 10^{\circ} \mathrm{C}\right)$ or pre-centrifuged $\left(4000 \mathrm{rpm}, \mathrm{RCF}=3800 \times g, 0.5 \mathrm{~h}, 10^{\circ} \mathrm{C}\right.$ Heraeus RS2.0 equipped with a 2258 swing-out rotor) to remove large $(>5 \mu \mathrm{m})$ mineral particles. Samples from low water conditions were not fractionated prior to investigation.

Lacustrine and marine samples were taken, in $\mathrm{HCl}$ cleaned, $\mathrm{H}_{2} \mathrm{O}$-rinsed, sterile polyethylene bottles, from Lake Lugano (Ticino, Switzerland) and from the Adriatic Sea off Rovinj (Croatia). Preparation of TEM specimens using ultracentrifugation was performed within $1 \mathrm{~h}$ after sampling. Subsets of marine samples 

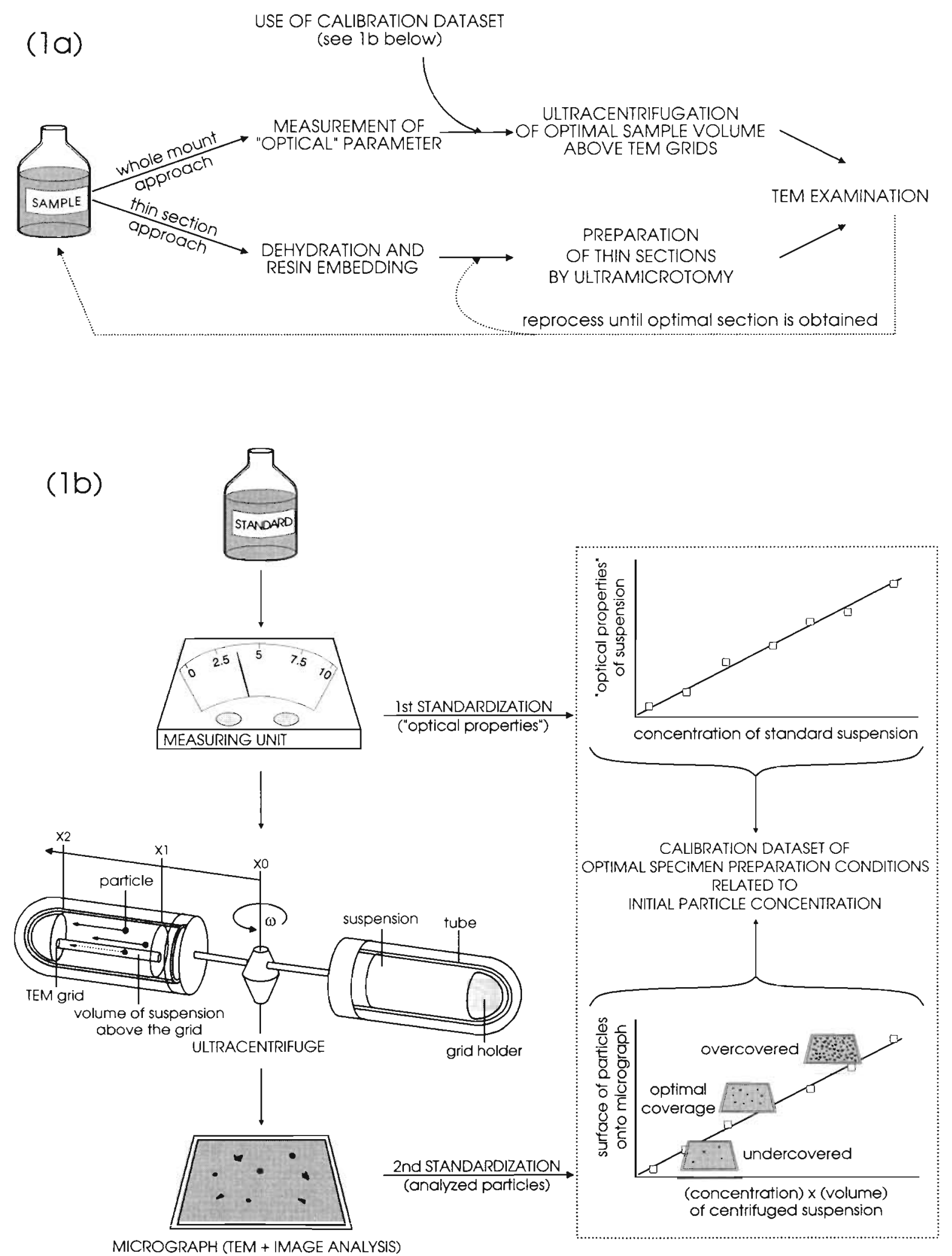

Fig. 1. (a) Specimen preparation scheme used in this study; (b) standardization of the whole mounts approach; the 'measuring unit' can be a fluorimeter, a turbidimeter or a light scattering spectrometer; the combination of the 1st and 2 nd calibration curves obtained with standard colloidal material allows the building of a calibration dataset which will be used for the preparation of specimens from unknown samples. TEM: transmission electron microscopy 
were also taken for preparation by classical resin embedding and ultramicrotomy; they were immediately fixed and stored at $4^{\circ} \mathrm{C}$ (see below).

Application of the whole mounts approach to natural colloids. The procedure developed for hematite microparticles was applied to the 3 river water samples (raw, sedimented, pre-centrifuged). In order to obtain the first calibration curve ('optical properties'; see Fig. 2), samples were diluted over a wide range $\left(1\right.$ to $\left.10^{3} \times\right)$ with synthetic electrolyte $\left[\mathrm{Ca}\left(\mathrm{HCO}_{3}\right)_{2} 1.5 \mathrm{mM}\right]$. As for hematite, volumes ranging between 2 and $20 \mathrm{ml}$ of these natural suspensions were centrifuged above TEM grids to build the second calibration curve (see Fig. 3).

Comparison of the set of calibration curves ('optical properties' vs particle concentration, Fig. 2; characteristics of particles vs mass of centrifuged particles, Fig. 3) and TEM images allows one to identify centrifugation conditions leading to the most appropriate micrographs (optimal loading of particles onto grids, without visual bias) for a given concentration of particles. The optimal volume of an unknown sample to be centrifuged above TEM grids can thus be determined by simply measuring its 'optical properties' and using the previously determined set of calibration curves.

Preparation of whole mounts by ultracentrifugation. Collodion-covered $(<10 \mathrm{~nm})$ carbon-coated $(<10 \mathrm{~nm})$ TEM copper grids (200 mesh) were used as specimen holders. Collection of particles was achieved by ultracentrifugation; turbidity- or light scattering-dependent volumes of suspensions were added to tubes mounted with flat grid holders (see Fig. 1b). The material was then deposited onto grids by ultracentrifugation. After centrifugation, the supernatant was carefully withdrawn and grids were recovered for post-treatment or direct TEM analysis. For marine samples, wet grids were immediately rinsed with $50 \mu$ of ultrapure water to avoid the artifactual formation of $\mathrm{NaCl}$ microcrystals

Fragile specimens can be protected with a thin layer of hydrophilic Nanoplast resin (Bachhuber \& Frösch 1983) to avoid shrinkage of thin fibrillar material. This is achieved by spinning a fresh Nanoplast mixture $\left(5 \mu \mathrm{l}_{i}\right.$ medium hardness resin diluted 1:10 with ultrapure water) on specimen grids mounted onto a horizontal-disk microcentrifuge (Perret et al. 1991). The resulting Nanoplast film is then polymerized and cured $\left(24 \mathrm{~h}\right.$ at room temperature, $24 \mathrm{~h}$ at $50^{\circ} \mathrm{C}$ ). Water rinsing to avoid formation of salt crystals is not necessary when grids are Nanoplast-protected.

Preparation of samples for the thin sections approach. For our correlative approach, and in order to monitor eventual artifacts caused by ultracentrifugation, we compared images derived from the whole mounts approach with samples prepared by classical resin embedding and ultrathin (50 to $70 \mathrm{~nm}$ ) sectioning methods
After fixation with glutaraldehyde (2.6\% final conc.) and ruthenium red $(0.05 \%$ final conc.) in Na-cacodylate- $\mathrm{HCl}$ buffer $(67 \mathrm{mM}$ final conc., $\mathrm{pH}=7$; Leppard et al. 1996), samples were post-fixed with $\mathrm{OsO}_{4}(1.33 \%$ final conc. with ruthenium red at $0.05 \%$ in cacodylate buffer) and dehydrated using a series of increasing methanol concentrations $(20,40,70,85,95,3 \times 100 \%$; Leppard et al. 1996). After dehydration, samples were embedded in Spurr's resin (Spurr 1969). Ultrathin sections were obtained with a diamond knife (Microstar Standard) using an MT-7 RMC ultramicrotome. Sections were placed on copper grids and examined by TEM after counterstaining.

Transmission electron microscopy. TEM observations were performed using a medium-resolution microscope (Zeiss EM10, $80 \mathrm{keV}$ ) for laboratory and freshwater preparations ( $\alpha-\mathrm{Fe}_{2} \mathrm{O}_{3}$, Arve River, Lake Lugano) and a high-resolution microscope (JEOL 1200 ExII TEMSCAN STEM, $80 \mathrm{keV}$ ) for marine samples.

\section{RESULTS AND DISCUSSION}

\section{Preparation of optimal TEM specimens by the whole mounts approach}

Validation of the calibration procedure, achieved with homodisperse hematite microparticles, is demonstrated in Figs. 2 \& 3. Fig. 2 shows the strong linear proportionality that exists between intensity of scattered light and concentration of hematite over a large range of environmentally relevant particle concentrations $\left(10^{1}\right.$ to $\left.10^{3} \mathrm{ug} \mathrm{l}^{-1}\right)$. The 'optical' response of colloids strongly depends on their physical characteristics (size, shape, refractive index). For instance, there is certainly no direct relationship in light scattering response between hematite microspheres and polysaccharide fibrils. It is thus expected that natural waters containing high proportions of mineral particles, or in opposition high proportions of natural organic colloids, will produce calibration curves with different slopes. This should be taken into account when samples of different origins are studied.

Fig. 3 exemplifies the second standardization step for hematitc. The total cross-section area of hematite particles measured on micrographs is strongly correlated to the mass of centrifuged particles $\left(10^{-1}\right.$ to $5 \times$ $10^{2} \mu$ g centrifuged), as expected. It can be seen from this example that optimal conditions for TEM examination (evenly dispersed hematite particles; insert 3a in Fig. 3) are achieved for a concentration of hematite close to $100 \mathrm{hg} \mathrm{l}^{-1}$ (light scattering intensity close to 20 in our case) and a centrifuged volume close to $4 \mathrm{ml}$, i.e. for a centrifuged mass of hematite close to $0.4 \mu \mathrm{g}$ (or $6.7 \times 10^{8}$ particles). This mass of hematite micro- 


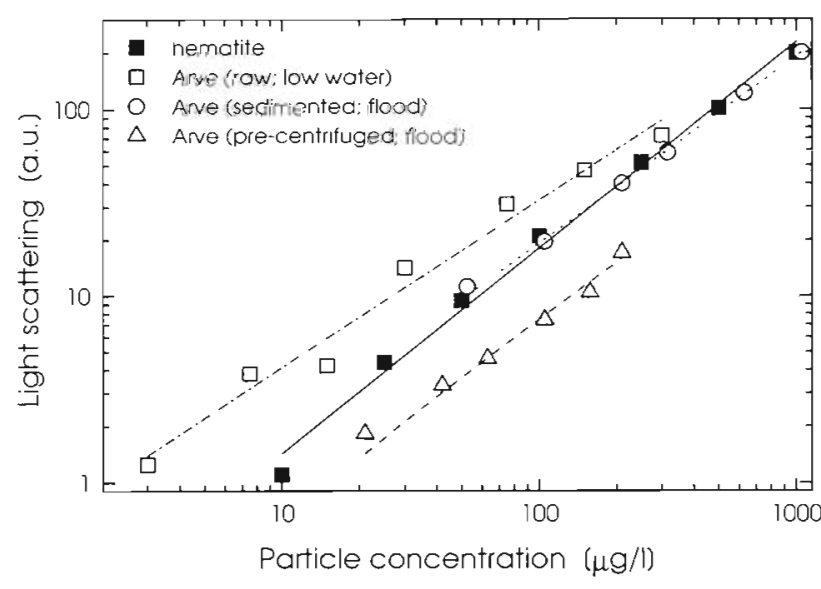

Fig. 2. Calibration curve of 'optical properties' of the suspension (in this case: intensity of scattered light; au: arbitrary units) versus mass concentration of the particles (standard hematite particles; Arve river water, Switzerland) in the suspension. In the case of the river samples fractionated by precentrifugation (flood event), the mass concentration was estimated to be less than $0.5 \%$ of the raw sample $\left(70 \mathrm{mg} \mathrm{l}^{-1}\right)$

particles in the initial suspension represents $1.4 \times 10^{8}$ particles $\mathrm{cm}^{-2}$ at the issue of the ultracentrifugation, for $r_{\text {tube }}=1.25 \mathrm{~cm}$ ( $r_{\text {tube }}=$ radius of tube $)$.

The same procedure was repeated for turbidity measurements; although turbidity is not directly related to light scattering, results were confirmed for hematite. Accordingly, river water samples were processed in a similar fashion in order to check the feasibility of the procedure for heterogeneous systems (see Figs. 2 \& 3); a tight correlation between cross-section area of particles on the final TEM images, and the initial characteristics of the natural suspensions, was also obtained

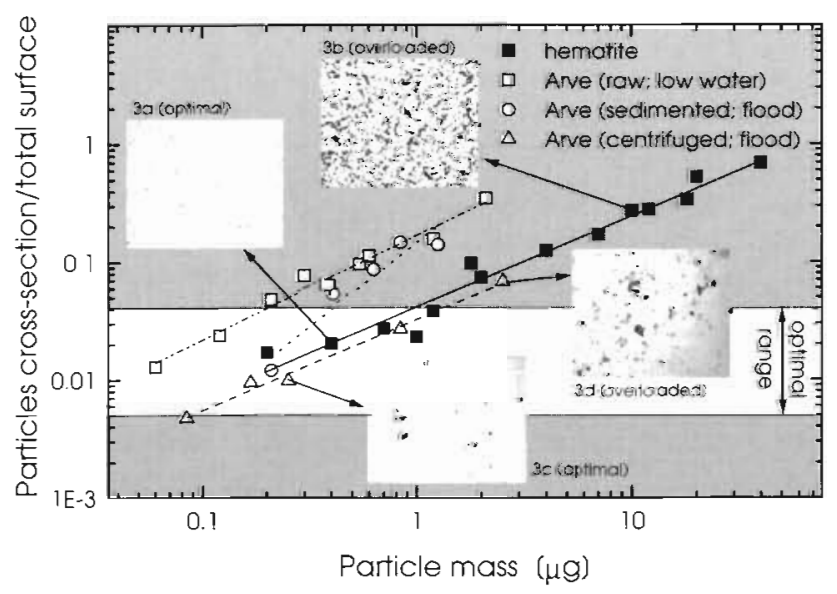

Fig. 3. Calibration curve of the characteristics of the particles (in this case: ratio of the cross-section of particles on the micrograph to the surface of the micrograph) versus mass of centrifuged particles (hematite particles; Arve river water). The particle mass is obtained by multiplying the concentration of particles with the centrifuged volume (inserts $3 c \& d$ in Fig. 3). The mass concentration of particles in the samples during the low water event (raw water: $3 \mathrm{mg} \mathrm{l}^{-1}$ ) and the flood event (raw water: $70 \mathrm{mg}^{1-1}$; scdimcnted watcr: $10.5 \mathrm{mg} \mathrm{l}^{-1}$ ) was obtained by filtration and weighing. However, in the case of the pre-centrifuged samples (flood event), attempts to determine the particle mass concentration in the supernatants failed because of high irreproducibility in the weighing of membranes and in filtration artifacts. We thus estimated that less than $0.5 \%$ (ca 200 to $350 \mathrm{\mu g} \mathrm{l}^{-1}$ ) of the total particle mass was retained in the samples after centrifugation (Perret et al. 1994).

The recovery of material onto TEM grids is a quantitative process when accurately selected conditions of centrifugation are chosen. The time (in seconds) required for sedimentation of particles of a given size and density to the bottom of a centrifuge tube can be calculated according to Stokes' law (see Fig. 1b):

$$
t_{\text {centrifugation }}=\left[18 \eta \ln \left(x_{2} / x_{1}\right)\right] /\left[d^{2}\left(\rho-\rho_{0}\right) \omega^{2}\right]
$$

where $\eta=$ viscosity of the solution (poise), $x_{2}$ and $x_{1}=$ maximum and minimum distances $(\mathrm{cm})$ in the tube relative to the axis of the centrifuge, $d=$ diameter of the colloid $(\mathrm{cm}), \rho$ and $\rho_{0}=$ densities $\left(\mathrm{g} \mathrm{cm}^{-3}\right)$ of the colloid and the solvent, $\omega=$ angular rotation speed of the centrifuge (radians $\mathrm{s}^{-1}$ )

For hematite, the yield of particle recovery onto TEM grids after ultracentrifugation was measured as $94 \%(\mathrm{CV}=8 \%$ for measurements made on TEM images containing 10 to 100 particles). Total recovery was also confirmed by the absence of microparticles in the supernatant. We observed however that long centrifugation times ( $>4 \mathrm{~h}$ ) induce losses of colloids by adsorption onto the walls of centrifugation tubes; this effect is non-negligible for waters with low particulate contents (pristine waters, groundwaters).

Provided the sample suspension is homogeneously dispersed, the final number of entities recovered onto TEM grids will be primarily determined by their initial number in the suspension and the volume to be centrifuged. Taking into account the characteristics of the centrifuge, the particles to be studied, and the analyzed surface on the TEM grid, the fraction $F$ of the grid covered by particles after centrifugation can be calculated according to:

$$
\begin{aligned}
F= & \text { (surface occupied by particles onto grid }) / \\
& (\text { surface of grid })=3[\text { particle }]\left(x_{2}-x_{1}\right) / 2 d \rho
\end{aligned}
$$

where $[$ particle] $=$ mass concentration of particles in the initial suspension $\left(\mathrm{g} \mathrm{cm}^{-3}\right)$. With hematite, the optimal value of $F$ for unbiased TEM examination appears to be between 0.005 and 0.05 , i.e. 0.5 to $5 \%$ of the surface of the grid is covered by microparticles.

The principle of the double calibration presented above allows one to obtain good estimates on the rela- 
tive concentration of particulate material in the suspension of interest, as exemplified by the riverine samples (see Figs. $2 \& 3$ ). The approach is quantitative and colloids are homogeneously dispersed onto the TEM grids. When suspensions of different origins present a large range of particle concentrations, they can be readily prepared under optimal conditions by finely tuning the volume to be centrifuged above TEM grids; it is thus possible to obtain 'optically' appropriate micrographs for groundwaters (low particulate content; large centrifuged volume) or for estuary samples (high particulate content; small centrifuged volume).

\section{Whole mounts versus thin sections: the correlative approach}

In order to avoid coalescence of fibrils surrounding bacteria during drying of grids, some specimens were coated with Nanoplast after ultracentrifugation to prevent dehydration. However, because of the mode of preparation of the protective film (Nanoplast spinning onto specimen grids), additional Nanoplast accumulates around large particles (thickness $>500 \mathrm{~nm}$ ), which leads to a poorer resolution. A decision must thus be taken between observation at medium resolution (after Nanoplast post-protection) and possible alteration of fibrils by drying effects (no protection) for samples containing large particulate material.

To enhance electron density of very fine organic material, the use of a stain is normally required. It has however been shown (Wilkinson et al. 1995, Lienemann et al. 1997) that staining procedures must be carefully undertaken to avoid precipitation of stain on fibrillar material. The optimal stain concentration obtained from different assays to clearly visualize exopolymers produced by bacteria is $0.1 \%$ uranyl acetate, but the reaction time strongly depends on the permeability of the specimen. Grids protected by Nanoplast required $20 \mathrm{~min}$ staining, while grids without Nanoplast were stained for $2 \mathrm{~min}$.

Artifacts produced by conventional preparation techniques (chemical fixation, dehydration, embedding into hydrophobic resin, ultramicrotomy) have been extensively studied in a correlative approach (Leppard et al. 1990, 1996, Leppard 1992b). The results obtained by a comparison of images derived from both embedding/ ultramicrotomy and ultracentrifugation, and the similarity in the structure of the exopolymers (see Fig. 4), suggest that artifacts are minimal for the latter method.

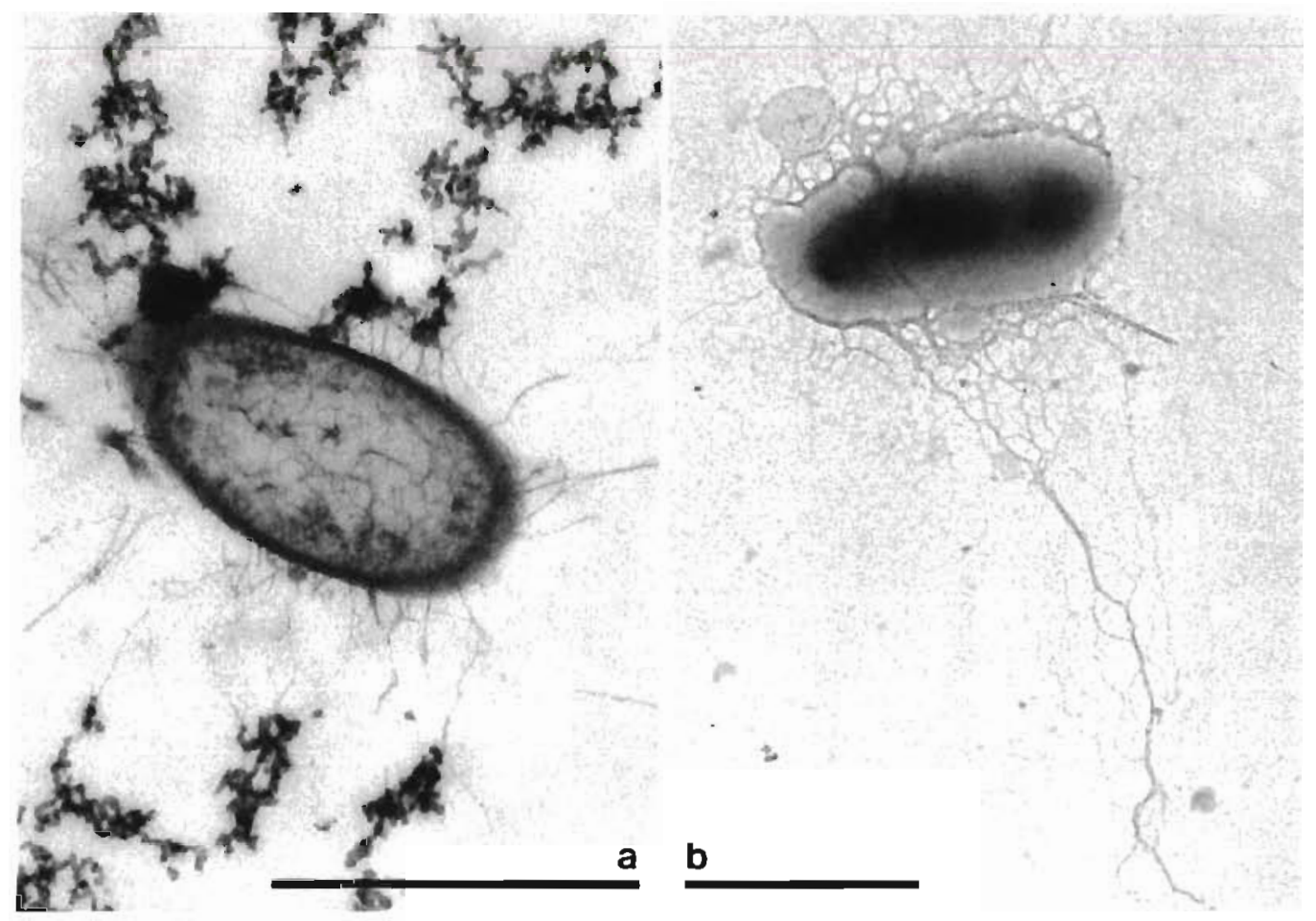

Fig. 4. (a) Ultrathin section (embedded in epoxy resin) of a marine sample (Adriatic Sea) showing fibrils produced by a bacterium; the thin section approach allows visualization of the internal differentiation of the bacterium. (b) Whole mount of a marine sample, prepared after optimal centrifugation, according to the procedure described in this paper; this specimen, which was Nanoplast post-protected, exhibits some fibrils as long continuous entities, as opposed to the short entities in thin sections. 
Information on the ultrastructure of fibrillar material which can be derived from images obtained by the 2 techniques is complementarily different. While the internal structure of cells can be optimally examined using ultramicrotomy, only segments of most fibrils are visualized in a given view, due to their native 3dimensional arrangement within the resin prior to ultramicrotomy (see Fig. 4a). Using centrifugation, whole exopolymer fibrils are visualized, therefore enabling measurement of their length and estimation of their extension from the bacterial cell wall into the water (see Fig. 4b). It has, however, to be stated that high centrifugation forces (e.g. RCF $=300000$ to $500000 \times g$ ) could result in partial removal of capsule from bacteria, especially when the capsular material is diffuse (e.g. extending outwards from a tightly aligned capsule) or loosely attached to the cell wall.

The whole mounts approach, when optimally processed, allows the rapid examination of associations between individual microorganisms, and between microorganisms and inorganic or organic particles (see Fig. 5). It can permit quantification of the number and kinds of attachment structures linking bacteria to other colloidal particles (e.g. minerals, organic debris, viruses, algae and other bacteria). Thus, an optimal whole mounts approach is a valuable new tool for costefficient analyses of particle bridging by fibrils, and by extracellular polymeric substances (EPS) in general, at least for small native associations.

In recent years, the formation and occurrence of transparent exopolymer particles (TEP) in marine systems has received considerable attention, because of the significance of TEP to ecophysiological processes (Alldredge et al. 1993, Passow et al. 1994, Passow \& Alldredge 1995). It is known that TEP are enriched in bacteria capable of producing significant amounts of colloidal EPS. This EPS is an essential factor for bacterial attachment to solid surfaces (Fletcher \& Floodgate 1973, Leppard 1993, Vandevivere \& Kirchman 1993), which in turn can affect many aquatic processes (Decho 1990). Part of the secreted EPS remains at the surface of the bacterial cells, creating a capsular envelope (colloid system) that may have a total volume (including its associated water) exceeding the cell volume manifold (Heissenberger et al. $1996 a, b)$. Considering the high relative abundance of these extensive extracellular capsules and the high number of bacteria in natural ecosystems $\left(10^{5}\right.$ to $10^{6} \mathrm{ml}^{-1}$ ), the EPS-rich bacteria might therefore influence production, structure and aggregation of the

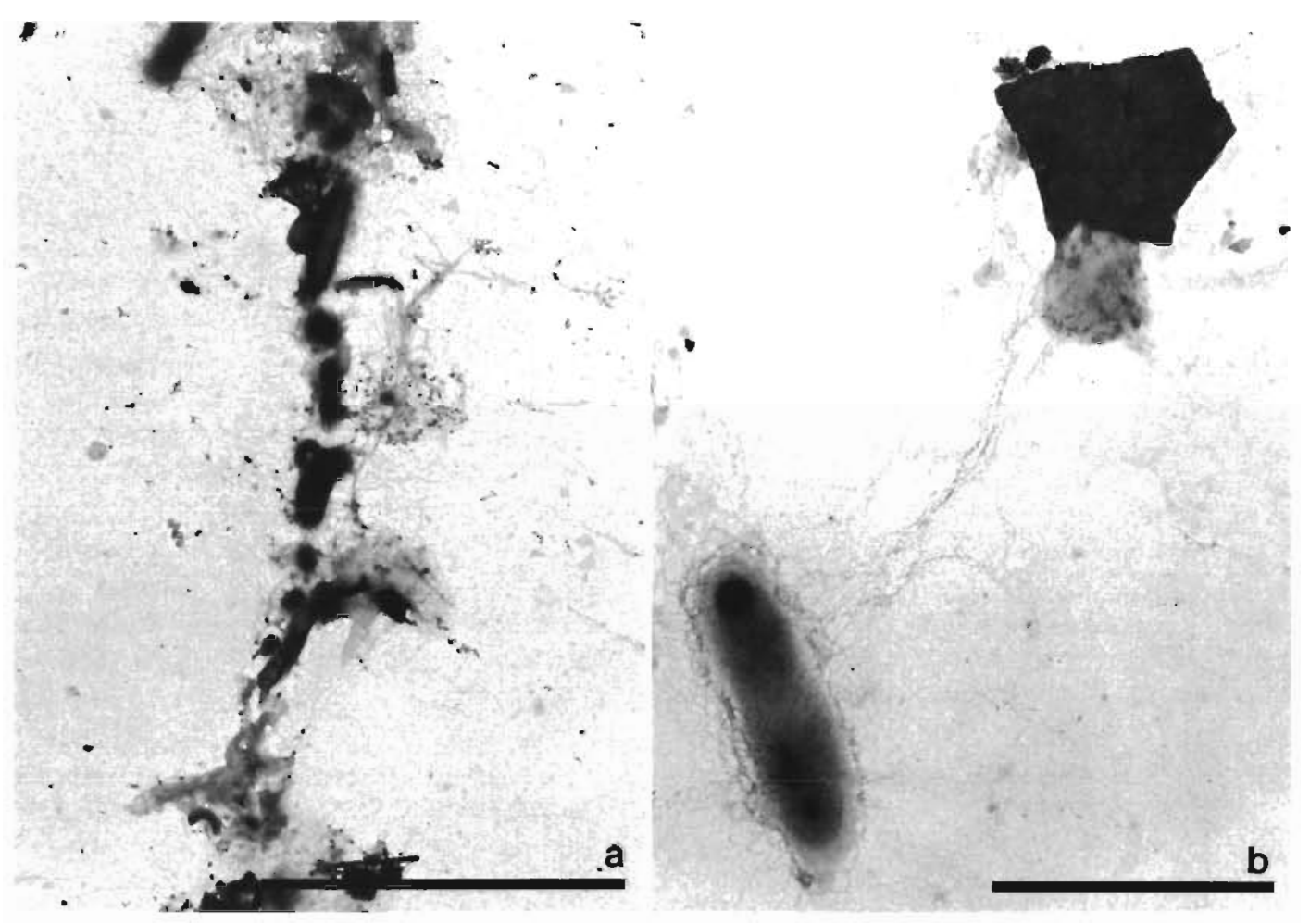

Fig. 5. Optimally prepared whole mount specimens from (a) Lake Lugano, Switzerland, sampled in a water layer enriched in autochthonous iron oxyhydroxide particles (scale bar $=4 \mu \mathrm{m}$ ), and (b) the Adriatic Sea, sampled during a marine snow event (scale bar $=1 \mu \mathrm{m})$. The complete integrity of fibrils within whole mounts allows one to reveal the intimate associations between microorganisms (a), and between microorganisms and mineral particles $(a, b)$ 
much larger TEP, as a result of high EPS sorptive capacities (Decho 1990). The relationships between EPS fibrils and the molecular architecture of TEP can be facilitated by the systematic correlative technology described here

In lakes and rivers, colloids play roles in the distribution of pollutants. This is a result of their sorptive capacities, great surface area relative to mass, and tendency to aggregate into particles subject to sedimentation (O'Melia 1985, Morel \& Gschwend 1987, Leppard 1993). Fibrillar EPS colloids (fibrils), as defined morphologically by TEM, have been recognized as abundant colloids with multiple roles (Leppard 1992a, 1993), including a role in bridging other colloids (Filella et al. 1993, Perret et al. 1994, Liss et al. 1996). Analysis of the native associations established by bridging, and the evolution of bridged colloids into flocs, is a subject (like marine TEP formation) which requires an optimal methodology for TEM preparation of water samples.

\section{CONCLUSION}

The whole mounts approach, consisting of centrifuging an optimal volume of suspension as a function of its 'optical properties', is a fast and efficient alternative to the thin sections approach. Whole mounts specimens are quantitative (i.e. they contain all colloids which, according to their size and density, must be deposited during the time of ultracentrifugation) and place all colloids in 1 focal plane for TEM examination, thus providing the equivalent of a large number of thin sections.

The results obtained by the whole mounts approach and by the thin sections approach are compatible and comparable. However, specimens prepared by ultracentrifugation (whole mounts approach) usually bring additional information when interactions between colloidal species are to be studied, because exopolymeric substances which are responsible for the attachment between colloids are recovered in their native fulllength state. The Iimitations on this approach for biological information are 2-fold: (1) non-rigid fibrils tend to lay flat in the focal plane, rather than forming the porous 3-dimensional network revealed by serial sections; (2) large aggregates (e.g. flocs) are not amenable to TEM analysis because they are too thick. Despite these limitations, ultracentrifuged small aggregates are readily employed to analyze the earliest stages of floc formation, including marine TEP and the abundant types of biota-rich flocs found in freshwater ecosystems.

This approach, which has proved to be effective for riverine, lacustrine and marine samples, could be applied to the study of colloidal interactions in other aquatic systems, provided care is taken to build calibration curves (Figs. 2 \& 3) with colloidal material having properties similar to the ones to be studied (small or large mineral particles, fibrillar exopolymers or humic-like material). Application of the proposed method to qualitative and quantitative evaluation of viruses could also bring additional information on these incompletely characterized species in natural waters.

Acknowledgements. We thank the staff of the Center for Marine Research in Rovinj (Croatia) for their hospitality and help during sampling, Prof. J. N. A. Lott (McMaster University, Hamilton, Canada) and Dr S. Fakan (University of Lausanne, Switzerland) for the use of electron microscopes, and M. M. West and D. T. Flannigan for preparing the sections. We are especially grateful to $G$. J. Herndl for financial support of A.H. This study has been made possible by a grant of the Swiss National Science Foundation to D.P. (\#20-42250.94).

\section{LITERATURE CITED}

Alldredge AL, Passow U, Logan BE (1993) The abundance and significance of a class of large, transparent organic particles in the ocean. Deep Sea Res I 40:1131-1140

Bachhuber K, Frösch D (1983) Melamine resins, a new class of water-soluble embedding media for electron microscopy. J Microsc 130:1-9

Buffle J, Leppard GG (1995) Characterization of aquatic colloids and macromolecules. 1. Structure and behavior of colloidal material. Environ Sci Technol 29:2169-2175

Decho AW (1990) Microbial exopolymer secretions in ocean environments: their role(s) in food webs and marine processes. Oceanogr Mar Biol Annu Rev 28:73-153

Filella M, Buffle J, Leppard GG (1993) Characterization of submicrometre colloids in freshwaters: evidence for their bridging by organic structures. Wat Sci Technol 27(11): $91-102$

Fletcher M, Floodgate GD (1973) An electron-microscopic demonstration of an acidic polysaccharide involved in the adhesion of a marine bacterium to solid surfaces. J Gen Microbiol 74:325-334

Heissenberger A, Leppard GG, HerndI GJ (1996a) Ultrastructure of marine snow: If. Microbiological considerations Mar Ecol Prog Ser 135:299-308

Heissenberger A, Leppard GG, Herndl GJ (1996b) Relationship between the intracellular integrity and the morphology of the capsular envelope in attached and free-living marine bacteria. Appl Environ Microbiol 62:4521-4528

Koike I, Hara S, Terauchi K, Kogure K (1990) Role of submicrometre particles in the ocean. Nature 345:242-244

Leppard GG (1992a) Evaluation of electron microscope techniques for the description of aquatic colloids. In: Buffle $J$, van Leeuwen HP (eds) Environmental particles, Vol 1 Lewis Publishers, Chelsea, MI, p 231-289

Leppard GG (1992b) Size, morphology and composition of particulates in aquatic ecosystems: solving speciation problems by correlative electron microscopy. Analyst 117 595-603

Leppard GG (1993) Organic flocs in surface waters - their native state and aggregation behavior in relation to contaminant dispersion. In: Rao SS (ed) Particulate matter 
and aquatic contaminants. Lewis Publishers, Chelsea, MI, p $169-195$

Leppard GG, Burnison BK, Buffle J (1990) Transmission electron microscopy of the natural organic matter of surface waters. Anal Chim Acta 232:107-121

Leppard GG, Heissenberger A, Herndl GJ (1996) Ultrastructure of marine snow: I. Transmission electron microscopy methodology. Mar Ecol Prog Ser 135:289-298

Leppard GG, West MM, Flannigan DT, Carson J, Lott JNA. (1997) A classification scheme for marine organic colloids in the Adriatic Sea: colloid speciation by transmission electron microscopy. Can J Fish Aquat Sci 54:2334-2349

Liang L (1988) Effects of surface chemistry on kinetics of coagulation of submicron iron oxide particles $\left(\alpha-\mathrm{Fe}_{2} \mathrm{O}_{3}\right)$ in water. PhD thesis, California lnstitute of Technology, Pasadena

Lienemann CP, Mavrocordatos D, Perret D (1997) Enhanced visualization of polysaccharides from aqueous suspensions. Mikrochim Acta 126:123-129

Liss SN, Droppo IG, Flannigan D, Leppard GG (1996) Floc architecture in wastewater and natural riverine systems. Environ Sci Technol 30:680-686

Matijevic E, Scheiner P (1978) Ferric hydrous oxide sols. III Preparation of uniform particles by hydrolysis of $\mathrm{Fe}(\mathrm{III})$ chloride, nitrate and perchlorate solutions. J Colloid Interface Sci 63:509-524

Mavrocordatos D, Perret D (1995) Non-artifacted specimen preparation for transmission electron microscopy of submicron soil particles. Commun Soil Sci Plant Anal 26 : 2593-2602

Morel FMM, Gschwend PM (1987) The role of colloids in the partitioning of solutes in natural waters. In: Stumm W (ed) Aquatic surface chemistry-chemical processes at the particle-water interface. John Wiley \& Sons, New York p 405-422

Editorial responsibility: Farooq Azam,

La Jolla, California, USA
Nomizu T, Mizuike A (1986) Electron microscopy of submicron particles in natural waters: specimen preparation by centrifugation. Mikrochim Acta 1:65-72

O'Melia CR (1985) The influence of coagulation and sedimentation on the fate of particles, associated pollutants and nutrients in lakes. In: Stumm W (ed) Chemical processes in lakes, Wiley-Interscience, New York, p 207-224

Passow U, Alldredge AL (1995) Aggregation of a diatom bloom in a mesocosm: the role of transparent exopolymer particles (TEP). Deep Sea Res II 42:99-109

Passow U, Alldredge AL, Logan BE (1994) The role of particulate carbohydrate exudates in the flocculation of diatom blooms. Deep Sea Res I 41:335-357

Perret D, Leppard GG, Müller M, Belzile N, DeVitre R, Buffle J (1991) Electron microscopy of aquatic colloids: non-perturbing preparation of specimens in the field. Water Res 25:1333-1343

Perret D, Newman ME, Nègre JC, Chen Y, Buffle J (1994) Submicron particles in the Rhine River. I. Physicochemical characterization. Water Res 28:91-106

Pizarro J, Belzile N, Filella M, Leppard GG, Nègre JC, Perret D, Buffle J (1995) Coagulation/sedimentation of submicron iron particles in a eutrophic lake. Water Res 29: $617-632$

Spurr AR (1969) A low-viscosity epoxy resin embedding medium for electron microscopy. J Ultrastruct Res 26:31-43

Vandevivere P, Kirchman DL (1993) Attachment stimulates exopolysaccharide synthesis by a bacterium. Appl Environ Microbiol 59:3280-3286

Wells ML, Goldberg ED (1992) Marine submicron particles. Mar Chem 40:5-18

Wilkinson KJ, Stoll S, Buffle J (1995) Characterization of NOM-colloid aggregates in surface waters: coupling transmission electron microscopy staining techniques and mathematical modelling. Fresenius J Anal Chem 351:54-61

Submitted: May 28, 1997; Accepted: September 6, 1997

Proofs received from author(s): January 5, 1998 